IMA Journal of Numerical Analysis (2016) Page 1 of 20

doi:10.1093/imanum/

\title{
Multilevel Interpolation of Divergence-Free Vector Fields
}

\author{
PATRICIO FARRELL $\dagger$ \\ Weierstrass Institute (WIAS), Mohrenstr. 39, 10117 Berlin, Germany
}

AND

KATHRYN GILLOW

Mathematical Institute, University of Oxford, Andrew Wiles Building, Radcliffe Observatory Quarter, Woodstock Road, Oxford, OX2 6GG, England

AND

HOLGER WENDLAND $\S$

Department of Mathematics, University of Bayreuth, 95440 Bayreuth, Germany

[Received on ; revised on ]

\begin{abstract}
We introduce a multilevel technique for interpolating scattered data of divergence-free vector fields with the help of matrix-valued compactly supported kernels. The support radius at a given level is linked to the mesh norm of the data set at that level. There are at least three advantages of this method: no grid structure is necessary for the implementation, the multilevel approach is computationally cheaper than solving a large one-shot system and the interpolant is guaranteed to be analytically divergence-free. Furthermore, though we will not pursue this here, our multilevel approach is able to represent multiple scales in the data if present. We will prove convergence of the scheme, stability estimates and give a numerical example. For the first time, we will also prove error estimates for derivatives and give approximation orders in terms of the fill distance of the finest data set.
\end{abstract}

Keywords: meshfree methods; multilevel approximation; divergence-free; radial basis functions.

\section{Introduction}

A vector field $\mathbf{v}: \Omega \subseteq \mathbb{R}^{d} \rightarrow \mathbb{R}^{d}$ is called divergence-free if it satisfies

$$
\operatorname{div}(\mathbf{v})=\nabla \cdot \mathbf{v}=0
$$

In fluid dynamics this condition is commonly used to model incompressible fluids. It is of great importance that this constraint is satisfied analytically. For example, when solving the magnetohydrodynamic equations, small numerical errors in the divergence constraint of the magnetic field $(\operatorname{div}(\mathbf{B})=0)$ lead to unstable and unphysical numerical solutions, see Brackbill \& Barnes (1980); McNally (2011).

Therefore, it is crucial to have discretisation techniques which provide analytically divergence-free approximations. One such technique is based upon matrix-valued kernels which have been investigated, for example, in Narcowich \& Ward (1994); Lowitzsch (2005b,c,a); Fuselier (2008c,b); Narcowich et al.

\footnotetext{
${ }^{\dagger}$ Email: patricio.farrell@wias-berlin.de

‡Email: kathryn.gillow@maths.ox.ac.uk

${ }^{\S}$ Corresponding author. Email: holger.wendland@uni-bayreuth.de
} 
(2007); Fuselier et al. (2009). These kernels have the additional advantage that they can approximate data at scattered nodes without needing to generate a mesh which becomes, especially for larger dimensions, prohibitively expensive.

Unfortunately, for a growing number of nodes one has to cope with increasing condition numbers and computational costs. Hence, in this paper we combine and analyse for the first time this matrixvalued approximation scheme with a recently investigated multilevel strategy (see for example Floater \& Iske (1996); Fasshauer (1999); Narcowich et al. (1999); Le Gia et al. (2010); Townsend \& Wendland (2013); Wendland (2010)), which reduces the computational cost significantly. The technique has the additional advantage of capturing different scales in the data if present.

This paper is organised as follows. In the next section, we introduce the necessary notation and background theory. In the third section we present the analytically divergence-free approximation algorithm, prove its convergence and analyse the condition numbers of the involved matrices. In the final section, we discuss a numerical example.

Though we concentrate on analytically divergence-free approximation spaces, our approach, including convergence and stability proofs, immediately carries over to analytically curl-free approximation spaces if designed similarly, see Fuselier (2008c).

\subsection{Notation}

For non-negative integer $k$ and $\Omega \subseteq \mathbb{R}^{d}$ let $H^{k}(\Omega)$ denote the Sobolev space with differentiability order $k$ and integrability power $p=2$. Define for $u \in H^{k}(\Omega)$ the Sobolev norms

$$
\|u\|_{H^{k}(\Omega)}^{2}:=\sum_{|\boldsymbol{\alpha}| \leqslant k}\left\|D^{\boldsymbol{\alpha}} u\right\|_{L_{2}(\Omega)}^{2} .
$$

For $\Omega=\mathbb{R}^{d}$ there is another way to characterise and generalise Sobolev spaces via Fourier transforms by defining

$$
H^{\sigma}\left(\mathbb{R}^{d}\right):=\left\{f \in L_{2}\left(\mathbb{R}^{d}\right) \mid \widehat{f}(\cdot)\left(1+\|\cdot\|_{2}^{2}\right)^{\sigma / 2} \in L_{2}\left(\mathbb{R}^{d}\right)\right\}
$$

where $0 \leqslant \sigma<\infty$ can now also denote a fractional positive number (Evans, 1998, Chapter 5). The norm on this space is naturally defined by

$$
\|f\|_{H^{\sigma}\left(\mathbb{R}^{d}\right)}^{2}:=(2 \pi)^{-d / 2} \int_{\mathbb{R}^{d}}|\widehat{f}(\boldsymbol{\omega})|^{2}\left(1+\|\boldsymbol{\omega}\|_{2}^{2}\right)^{\sigma} d \boldsymbol{\omega},
$$

which is equivalent to the norm (1.2) in the case of $\sigma=k \in \mathbb{N}_{0}$. Here, the Fourier transform of an integrable function $f \in L_{1}\left(\mathbb{R}^{d}\right)$ is defined to be

$$
\widehat{f}(\boldsymbol{\omega}):=(2 \pi)^{-d / 2} \int_{\mathbb{R}^{d}} f(\mathbf{x}) e^{-i \mathbf{x}^{T} \boldsymbol{\omega}} d \mathbf{x}, \quad \boldsymbol{\omega} \in \mathbb{R}^{d}
$$

and then extended to $L_{2}\left(\mathbb{R}^{d}\right)$-functions in the usual way.

We will also use fractional order Sobolev spaces on bounded domains, i.e. $H^{\sigma}(\Omega)$ with $\sigma \geqslant 0$, which can, for example, be introduced by interpolation between integer order Sobolev spaces. Further function spaces will be introduced later on.

\section{Positive Definite Matrix-Valued Kernels}

A continuous scalar-valued function $\phi: \mathbb{R}^{d} \rightarrow \mathbb{R}$ is called positive semi-definite on $\mathbb{R}^{d}$ if for all $N \in \mathbb{N}$, any finite set $X=\left\{\mathbf{x}_{1}, \mathbf{x}_{2}, \ldots, \mathbf{x}_{N}\right\} \subseteq \mathbb{R}^{d}$ of pairwise distinct points, and all $\boldsymbol{\alpha}=\left(\alpha_{1}, \ldots, \alpha_{N}\right)^{T} \in \mathbb{R}^{N}$, the 
quadratic form

$$
\sum_{j=1}^{N} \sum_{k=1}^{N} \alpha_{j} \alpha_{k} \phi\left(\mathbf{x}_{j}-\mathbf{x}_{k}\right)
$$

is non-negative. It is called positive definite on $\mathbb{R}^{d}$ if the quadratic form is positive for all $\boldsymbol{\alpha} \in \mathbb{R}^{N} \backslash\{\boldsymbol{0}\}$.

We will be concerned with compactly supported radial basis functions for which the Fourier transform exhibits algebraic decay. The basis function $\phi: \mathbb{R}^{d} \rightarrow \mathbb{R}$ is radial if it is of the form $\phi(\mathbf{x})=$ $\phi_{0}\left(\|\mathbf{x}\|_{2}\right)$, where $\|\cdot\|_{2}$ denotes the Euclidean norm on $\mathbb{R}^{d}$. The Fourier transform of an integrable function $\phi$ decays algebraically, if there are two constants $c_{1}, c_{2}>0$ and some $\tau>d / 2$ such that

$$
c_{1}\left(1+\|\boldsymbol{\omega}\|_{2}^{2}\right)^{-\tau} \leqslant \widehat{\phi}(\boldsymbol{\omega}) \leqslant c_{2}\left(1+\|\boldsymbol{\omega}\|_{2}^{2}\right)^{-\tau} .
$$

Typical examples are given in Johnson (2012); Schaback (2011); Wendland (1995, 2005).

In this section, we introduce positive definite matrix-valued kernels, which we will eventually use to construct divergence-free approximants. The theory for positive definite matrix-valued kernels was originally developed by Narcowich and Ward in Narcowich \& Ward (1994). We will follow an approach by Fuselier (Fuselier $(2008 \mathrm{c}, \mathrm{b})$ ) for introducing the theory, which mimics some of the ideas from the scalar-valued case, see Wendland (2005).

Let us start by making a reasonable generalisation of positive definite scalar-valued kernels.

Definition 2.1 A continuous matrix-valued kernel $\Phi: \mathbb{R}^{d} \rightarrow \mathbb{R}^{d \times d}$ is called positive definite if it is even, $\Phi(-\mathbf{x})=\Phi(\mathbf{x})$, symmetric, $\Phi(\mathbf{x})=\Phi(\mathbf{x})^{T}$, and if

$$
\sum_{j, k=1}^{N} \boldsymbol{\alpha}_{j}^{T} \Phi\left(\mathbf{x}_{j}-\mathbf{x}_{k}\right) \boldsymbol{\alpha}_{k}>0
$$

for all $N \in \mathbb{N}$, all pairwise distinct $\mathbf{x}_{j} \in \mathbb{R}^{d}$ and all $\boldsymbol{\alpha}_{j} \in \mathbb{R}^{d}$, not all of them vanishing.

In this paper, we will exclusively be interested in one specific example of a matrix-valued positive definite kernel. Let $\phi: \mathbb{R}^{d} \rightarrow \mathbb{R}$ be a positive definite function in $C^{2}\left(\mathbb{R}^{d}\right)$. Then we define

$$
\Phi: \mathbb{R}^{d} \rightarrow \mathbb{R}^{d \times d}, \quad \Phi:=\left(-\Delta I+\nabla \nabla^{T}\right) \phi
$$

where $\Delta$ is the Laplacian, $\nabla$ the gradient and $I$ the $d$-dimensional identity matrix. The component-wise Fourier transform is given by

$$
\widehat{\Phi}(\boldsymbol{\omega})=\left(\|\boldsymbol{\omega}\|_{2}^{2} I-\omega \omega^{T}\right) \widehat{\phi}(\boldsymbol{\omega}) .
$$

For just two space dimensions the kernel $\Phi$ takes the form

$$
\Phi=\left(\begin{array}{cc}
-\partial_{22} & \partial_{12} \\
\partial_{21} & -\partial_{11}
\end{array}\right) \phi=\left(\begin{array}{cc}
-\frac{x_{2}^{2}}{r^{2}} \phi_{0}^{\prime \prime}(r)-\frac{x_{1}^{2}}{r^{3}} \phi_{0}^{\prime}(r) & \frac{x_{1} x_{2}}{r^{2}} \phi_{0}^{\prime \prime}(r)-\frac{x_{1} x_{2}}{r^{3}} \phi_{0}^{\prime}(r) \\
\frac{x_{1} x_{2}}{r^{2}} \phi_{0}^{\prime \prime}(r)-\frac{x_{1} x_{2}}{r^{3}} \phi_{0}^{\prime}(r) & -\frac{x_{1}^{2}}{r^{2}} \phi_{0}^{\prime \prime}(r)-\frac{x_{2}^{2}}{r^{3}} \phi_{0}^{\prime}(r)
\end{array}\right)
$$

provided $\phi$ is radial with $\phi(\mathbf{x})=\phi_{0}(r)$ where $r=\sqrt{x_{1}^{2}+x_{2}^{2}}$. Therefore, in general matrix-valued kernels are not radial. Nevertheless, they are commonly referred to as matrix-valued radial basis functions. By construction the kernel $\Phi$ consists of divergence-free columns and rows. It is possible to show that it is indeed positive definite in the sense of Definition 2.1. The following result essentially follows from the general theory in Narcowich \& Ward (1994). 
THEOREM 2.2 Let $\phi \in W_{1}^{2}\left(\mathbb{R}^{d}\right) \cap C^{2}\left(\mathbb{R}^{d}\right)$ be positive definite. Then the kernel $\Phi$ is positive definite.

As usual, the space $W_{1}^{2}\left(\mathbb{R}^{d}\right)$ consists of all functions $u$ which are, together with their weak derivatives up to order two, in $L_{1}\left(\mathbb{R}^{d}\right)$.

\subsection{Reproducing Kernel Hilbert spaces}

In the case of classical positive definite functions $\phi: \mathbb{R}^{d} \rightarrow \mathbb{R}$ it is well-known that associated to these functions is a Hilbert space, called the native space of $\phi$, in which $\phi$ acts as the reproducing kernel. This Hilbert space can be defined by completing the shifts of the basis function with respect to an inner product defined by the positive definite function (see for example Wendland (1995)).

The same can be done for matrix-valued kernels $\Phi: \mathbb{R}^{d} \rightarrow \mathbb{R}^{d \times d}$ (see for example Fuselier (2008c); Wendland (2005)) and leads to a Hilbert space $\mathscr{N}_{\Phi}\left(\mathbb{R}^{d}\right)$ of vector-valued functions $\mathbf{f}: \mathbb{R}^{d} \rightarrow \mathbb{R}^{d}$, which is a reproducing kernel Hilbert space in the sense that for $\mathbf{f} \in \mathscr{N}_{\Phi}\left(\mathbb{R}^{d}\right), \boldsymbol{\alpha} \in \mathbb{R}^{d}$ and $\mathbf{x} \in \mathbb{R}^{d}$ we have

$$
\Phi(\cdot-\mathbf{x}) \boldsymbol{\alpha} \in \mathscr{N}_{\Phi}\left(\mathbb{R}^{d}\right), \quad(\mathbf{f}, \Phi(\cdot-\mathbf{x}) \boldsymbol{\alpha})_{\Phi}=\mathbf{f}(\mathbf{x})^{T} \boldsymbol{\alpha} .
$$

In the case of our divergence-free kernels (2.3), there is a useful characterisation for such native spaces. A proof of the following result can be found in (Fuselier, 2008c, Theorem 2 and 3) as well as in (Wendland, 2009, Theorem 3.4).

THEOREM 2.3 Suppose the positive definite kernel $\phi$ lies in $W_{1}^{2}\left(\mathbb{R}^{d}\right) \cap C^{2}\left(\mathbb{R}^{d}\right)$. Then the native space of the divergence-free kernel is given by

$$
\mathscr{N}_{\Phi}\left(\mathbb{R}^{d}\right)=\left\{\mathbf{f} \in \mathbf{L}_{2}\left(\mathbb{R}^{d}\right) \cap \mathbf{C}\left(\mathbb{R}^{d}\right) \mid\|\mathbf{f}\|_{\mathscr{N}_{\Phi}\left(\mathbb{R}^{d}\right)}<\infty, \operatorname{div}(\mathbf{f})=0\right\}
$$

where the native space norm is given by

$$
\|\mathbf{f}\|_{\mathscr{N}_{\Phi}\left(\mathbb{R}^{d}\right)}^{2}=(2 \pi)^{-d / 2} \int_{\mathbb{R}^{d}} \frac{\|\widehat{\mathbf{f}}(\boldsymbol{\omega})\|_{2}^{2}}{\|\boldsymbol{\omega}\|_{2}^{2} \widehat{\phi}(\boldsymbol{\omega})} d \boldsymbol{\omega} .
$$

Here $\mathbf{L}_{2}\left(\mathbb{R}^{d}\right)$ and $\mathbf{C}\left(\mathbb{R}^{d}\right)$ denote vector-valued function spaces with each component in $L_{2}\left(\mathbb{R}^{d}\right)$ or $C\left(\mathbb{R}^{d}\right)$, respectively.

\subsection{Native Spaces as Sobolev Spaces}

As in the scalar-valued case, it is possible to interpret the native space under some assumptions as some type of Sobolev space. For scalar-valued kernels it is well-known that the native space of a scalar-valued function is norm-equivalent to a classical Sobolev space if the Fourier transform of the kernel has an algebraic decay of the form (2.2), see Wendland (2005).

We define the vector-valued Sobolev space $\mathbf{H}^{\sigma}(\Omega)$ to consist of functions $\mathbf{u}=\left(u_{1}, \ldots, u_{d}\right)^{T}: \Omega \rightarrow$ $\mathbb{R}^{d}$ for which each component lies in the scalar-valued Sobolev space $H^{\sigma}(\Omega)$. A norm on the vectorvalued Sobolev space can be defined with the help of the scalar-valued Sobolev norm in the following way

$$
\|\mathbf{u}\|_{\mathbf{H}^{\sigma}(\Omega)}:=\left(\sum_{j=1}^{d}\left\|u_{j}\right\|_{H^{\sigma}(\Omega)}^{2}\right)^{1 / 2} .
$$


We introduce divergence-free Sobolev spaces,

$$
\mathbf{H}^{\sigma}\left(\mathbb{R}^{d}, \operatorname{div}\right):=\left\{\mathbf{f} \in \mathbf{H}^{\sigma}\left(\mathbb{R}^{d}\right) \mid \operatorname{div}(\mathbf{f})=0\right\}
$$

With the help of these spaces we define subspaces

$$
\begin{aligned}
\widetilde{\mathbf{H}}^{\sigma}\left(\mathbb{R}^{d}\right) & :=\left\{\mathbf{f} \in \mathbf{H}^{\sigma}\left(\mathbb{R}^{d}\right) \mid\|\mathbf{f}\|_{\widetilde{\mathbf{H}}^{\sigma}\left(\mathbb{R}^{d}\right)}<\infty\right\}, \\
\widetilde{\mathbf{H}}^{\sigma}\left(\mathbb{R}^{d}, \operatorname{div}\right) & :=\left\{\mathbf{f} \in \mathbf{H}^{\sigma}\left(\mathbb{R}^{d}, \operatorname{div}\right) \mid\|\mathbf{f}\|_{\widetilde{\mathbf{H}}^{\sigma}\left(\mathbb{R}^{d}\right)}<\infty\right\},
\end{aligned}
$$

where

$$
\|\mathbf{f}\|_{\widetilde{\mathbf{H}}^{\sigma}\left(\mathbb{R}^{d}\right)}^{2}:=(2 \pi)^{-d / 2} \int_{\mathbb{R}^{d}} \frac{\|\widehat{\mathbf{f}}(\boldsymbol{\omega})\|_{2}^{2}}{\|\boldsymbol{\omega}\|_{2}^{2}}\left(1+\|\boldsymbol{\omega}\|_{2}^{2}\right)^{\sigma+1} d \boldsymbol{\omega} .
$$

All the spaces introduced in this section are obviously related. Let us consider a divergence-free velocity field $\mathbf{u}$, which lies in the space $\widetilde{\mathbf{H}}^{\sigma}\left(\mathbb{R}^{d}\right.$, div) for some $\sigma>0$. This space, however, is a subspace of $\mathbf{H}^{\sigma}\left(\mathbb{R}^{d}\right)$. Therefore, we may sometimes write $\mathbf{u}$ in the $\mathbf{H}^{\sigma}\left(\mathbb{R}^{d}\right)$ norm as well. Moreover, for $\mathbf{u} \in \widetilde{\mathbf{H}}^{\sigma}\left(\mathbb{R}^{d}\right)$ we obviously have

$$
\|\mathbf{u}\|_{\mathbf{H}^{\sigma}\left(\mathbb{R}^{d}\right)}^{2} \leqslant\|\mathbf{u}\|_{\widetilde{\mathbf{H}}^{\sigma}\left(\mathbb{R}^{d}\right)}^{2}
$$

showing particularly that the inclusions $\widetilde{\mathbf{H}}^{\sigma}(\mathbb{R})^{d} \subseteq \mathbf{H}^{\sigma}\left(\mathbb{R}^{d}\right)$ and also $\widetilde{\mathbf{H}}^{\sigma}\left(\mathbb{R}^{d}, \mathrm{div}\right) \subseteq \mathbf{H}^{\sigma}\left(\mathbb{R}^{d}\right.$, div $)$ are continuous.

Now we are able to state another characterisation of the native spaces that we have discussed so far. To this end, note that if $\phi \in L_{1}\left(\mathbb{R}^{d}\right)$ has a Fourier transform satisfying (2.2) with $\tau=\sigma+1$ and $\sigma>d / 2$ then $\phi \in C^{2}\left(\mathbb{R}^{d}\right)$, though the Sobolev embedding theorem only yields $H^{\sigma}\left(\mathbb{R}^{d}\right) \subseteq C^{1}\left(\mathbb{R}^{d}\right)$. This can be seen as follows. Under the assumptions, we can invoke the Fourier inversion formula to write

$$
\phi(\mathbf{x})=(2 \pi)^{-d / 2} \int_{\mathbb{R}^{d}} \widehat{\phi}(\boldsymbol{\omega}) e^{i \mathbf{x}^{T} \boldsymbol{\omega}} d \boldsymbol{\omega} .
$$

We can differentiate under the integral

$$
D^{\alpha} \phi(\mathbf{x})=(2 \pi)^{-d / 2} \int_{\mathbb{R}^{d}} \widehat{\phi}(\boldsymbol{\omega})(i \boldsymbol{\omega})^{\alpha} e^{i \mathbf{x}^{T} \boldsymbol{\omega}} d \boldsymbol{\omega},
$$

as long as the function $\boldsymbol{\omega} \mapsto \widehat{\phi}(\boldsymbol{\omega})(i \boldsymbol{\omega})^{\alpha}$ belongs to $L_{1}\left(\mathbb{R}^{d}\right)$. This function is continuous and for second order derivatives, i.e. $|\alpha|=2$, it behaves for large $\boldsymbol{\omega}$ like $\|\boldsymbol{\omega}\|_{2}^{-2 \sigma-2+|\alpha|}=\|\boldsymbol{\omega}\|_{2}^{-2 \sigma}$, showing integrability as long as $\sigma>d / 2$.

THEOREM 2.4 Let $\sigma>d / 2$. Let $\phi$ generate $H^{\sigma+1}\left(\mathbb{R}^{d}\right)$ as its native space, i.e. $\phi \in L_{1}\left(\mathbb{R}^{d}\right)$ possesses a Fourier transform satisfying (2.2) with $\tau=\sigma+1$. Then, the native space of the matrix-valued kernel $\Phi=\left(-\Delta I+\nabla \nabla^{T}\right) \phi$ is given by

$$
\mathscr{N}_{\Phi}\left(\mathbb{R}^{d}\right)=\widetilde{\mathbf{H}}^{\sigma}\left(\mathbb{R}^{d}, \operatorname{div}\right) .
$$

The proof follows immediately from Theorem 2.3. For details, see Fuselier (2008c); Wendland (2009).

We finish this section by introducing a vector-valued extension operator since we are mostly interested in bounded domains and therefore need to extend our locally defined Sobolev functions to functions defined on the whole Euclidean space. The following result follows from (Wendland, 2009, Proposition 3.8.) by setting the pressure component to zero. 
Proposition 2.5 Suppose either $d=2$ or $d=3$. Let $\sigma \geqslant 0$ and let $\Omega \subseteq \mathbb{R}^{d}$ be a simply-connected domain with $C^{k, 1}$ boundary, where $k \geqslant \sigma$ is some integer. Then there exists a continuous operator

$$
\mathbf{E}_{\text {div }}: \mathbf{H}^{\tau}(\Omega, \operatorname{div}) \rightarrow \widetilde{\mathbf{H}}^{\tau}\left(\mathbb{R}^{d}, \operatorname{div}\right), \quad 0 \leqslant \tau \leqslant \sigma,
$$

such that

1. $\left.\left(\mathbf{E}_{\mathrm{div}} \mathbf{v}\right)\right|_{\Omega}=\left.\mathbf{v}\right|_{\Omega}$

2. $\left\|\mathbf{E}_{\text {div }} \mathbf{v}\right\|_{\widetilde{\mathbf{H}}^{\tau}\left(\mathbb{R}^{d}, \text { div }\right)} \leqslant C\|\mathbf{v}\|_{\mathbf{H}^{\tau}(\Omega, \operatorname{div})}$,

for all $\mathbf{v} \in \mathbf{H}^{\tau}(\Omega, \operatorname{div})$, with some $C=C_{\tau}>0$.

Finally, we need a lemma from Chernih \& Gia (2013). For the convenience of the reader we include its proof here.

Lemma 2.1 Under the assumptions of Proposition 2.5 let $\mathbf{u} \in \mathbf{H}^{\sigma}(\Omega$, div $)$ with $\sigma>0$. Then

$$
\int_{\mathbb{R}^{d}} \frac{\left\|\widehat{\mathbf{E}_{\mathrm{div}}} \mathbf{u}(\boldsymbol{\omega})\right\|_{2}^{2}}{\|\boldsymbol{\omega}\|_{2}^{2}} d \boldsymbol{\omega} \leqslant C\|\mathbf{u}\|_{\mathbf{L}_{2}(\Omega)}^{2} .
$$

Proof. To prove this result for $d=3$, we need to discuss the extension operator $\mathbf{E}_{\mathrm{div}}$ in more detail. This operator involves the standard, bounded extension operator $E_{S}: H^{\sigma}(\Omega) \rightarrow H^{\sigma}\left(\mathbb{R}^{d}\right)$ as well a specifically constructed, bounded operator $T: \mathbf{H}^{\sigma}(\Omega, \operatorname{div}) \rightarrow \mathbf{H}^{\sigma+1}(\Omega)$ which satisfies

$$
\mathbf{u}=\nabla \times T \mathbf{u}
$$

for all $\mathbf{u} \in \mathbf{H}^{\sigma}(\Omega, \operatorname{div})$. Then $\mathbf{E}_{\text {div }}$ is defined as

$$
\mathbf{E}_{\text {div }}(\mathbf{u})=\nabla \times E_{S} T \mathbf{u},
$$

where $E_{S}$ is taken component-wise. For more details on the construction of this operator, we refer to Wendland (2009). Using this definition, we compute

$$
\begin{aligned}
\int_{\mathbb{R}^{d}} \frac{\left\|\widehat{\mathbf{E}_{\mathrm{div}} \mathbf{u}}(\boldsymbol{\omega})\right\|_{2}^{2}}{\|\boldsymbol{\omega}\|_{2}^{2}} d \boldsymbol{\omega} & =\int_{\mathbb{R}^{d}} \frac{\left\|\boldsymbol{\omega} \times \widehat{E_{S} T \mathbf{u}}(\boldsymbol{\omega})\right\|_{2}^{2}}{\|\boldsymbol{\omega}\|_{2}^{2}} d \boldsymbol{\omega} \leqslant C \int_{\mathbb{R}^{d}}\left\|\widehat{E_{S} T \mathbf{u}}(\boldsymbol{\omega})\right\|_{2}^{2} d \boldsymbol{\omega} \\
& \leqslant C\left\|E_{S} T \mathbf{u}\right\|_{\mathbf{L}_{2}\left(\mathbb{R}^{d}\right)}^{2} \leqslant C\left\|E_{S} T \mathbf{u}\right\|_{\mathbf{H}^{1}\left(\mathbb{R}^{d}\right)}^{2} \\
& \leqslant C\|T \mathbf{u}\|_{\mathbf{H}^{1}(\Omega)}^{2} \leqslant C\|\mathbf{u}\|_{\mathbf{L}_{2}(\Omega)}^{2} .
\end{aligned}
$$

\subsection{Reconstruction Problem}

The following discussion is well-known from the theory of scalar-valued kernels which can easily be extended to matrix-valued kernels (Wendland (2009)). Hence, we only give a short review of some of the key results. Suppose we want to reconstruct data $\mathbf{f}_{1}, \ldots, \mathbf{f}_{N} \in \mathbb{R}^{d}$ at scattered data points $X=$ $\left\{\mathbf{x}_{1}, \ldots, \mathbf{x}_{N}\right\} \subseteq \mathbb{R}^{d}$. For some coefficient vectors $\boldsymbol{\alpha}_{j} \in \mathbb{R}^{d}$, we can set up a vector-valued interpolant of the form

$$
\mathbf{S}_{\mathbf{f}, X}:=\sum_{j=1}^{N} \Phi\left(\cdot-\mathbf{x}_{j}\right) \boldsymbol{\alpha}_{j}
$$


and apply to it the interpolation conditions

$$
\mathbf{s}_{\mathbf{f}, X}\left(\mathbf{x}_{k}\right)=\mathbf{f}_{k}
$$

for $k=1, \ldots, N$. This means we have to solve the $d N \times d N$ block matrix system

$$
\left(\begin{array}{ccc}
\Phi\left(\mathbf{x}_{1}-\mathbf{x}_{1}\right) & \ldots & \Phi\left(\mathbf{x}_{1}-\mathbf{x}_{N}\right) \\
\vdots & & \vdots \\
\Phi\left(\mathbf{x}_{N}-\mathbf{x}_{1}\right) & \ldots & \Phi\left(\mathbf{x}_{N}-\mathbf{x}_{N}\right)
\end{array}\right)\left(\begin{array}{c}
\boldsymbol{\alpha}_{1} \\
\vdots \\
\boldsymbol{\alpha}_{N}
\end{array}\right)=\left(\begin{array}{c}
\mathbf{f}_{1} \\
\vdots \\
\mathbf{f}_{N}
\end{array}\right)
$$

or in abbreviated form

$$
A_{\Phi, X} \boldsymbol{\alpha}=\mathbf{f} .
$$

Note that for $\boldsymbol{\beta}=\left(\boldsymbol{\beta}_{1}, \ldots, \boldsymbol{\beta}_{N}\right)^{T} \in \mathbb{R}^{d N}$, we have

$$
\boldsymbol{\beta}^{T} A_{\Phi, X} \boldsymbol{\beta}=\sum_{j, k=1}^{N} \boldsymbol{\beta}_{j}^{T} \Phi\left(\mathbf{x}_{j}-\mathbf{x}_{k}\right) \boldsymbol{\beta}_{k}>0,
$$

since $\Phi$ is a positive definite kernel. Therefore, the matrix $A_{\Phi, X}$ is positive definite and the interpolant (2.6) with coefficient vectors determined by (2.8) exists and is unique.

Assuming the data are generated by $\mathbf{f}_{j}=\mathbf{f}\left(\mathbf{x}_{j}\right)$ with $\mathbf{f} \in \mathscr{N}_{\Phi}\left(\mathbb{R}^{d}\right)$, the interpolant $\mathbf{s}_{\mathbf{f}, X}$ is also the best approximation from

$$
\mathbf{V}_{X}=\left\{\sum_{j=1}^{N} \Phi\left(\cdot-\mathbf{x}_{j}\right) \boldsymbol{\beta}_{j} \mid \boldsymbol{\beta}_{j} \in \mathbb{R}^{n}\right\},
$$

to $\mathbf{f}$ in the native space norm. This is well-known for the scalar-valued case and follows here in the same fashion from the reproducing kernel propery (2.4). This best approximation property immediately implies two stability results. Namely,

$$
\left\|\mathbf{f}-\mathbf{s}_{\mathbf{f}, X}\right\|_{\Phi} \leqslant\|\mathbf{f}\|_{\Phi} \quad \text { and } \quad\left\|\mathbf{s}_{\mathbf{f}, X}\right\|_{\Phi} \leqslant\|\mathbf{f}\|_{\Phi} .
$$

In theory, we could use this idea to interpolate divergence-free fields with a divergence-free kernel. However, just as in the scalar-valued kernel case, the condition number of the linear system (2.8) will become very large when interpolating many nodes. Also, the density of the matrices and the resulting computational cost limits the number of points, particularly, since our system now has dimension $d N \times$ $d N$ instead of $N \times N$ in the scalar-valued case. This motivates our multilevel idea in the next section.

\section{Multilevel Theory for Divergence-Free Vector Fields}

We define scaled kernels via

$$
\Phi_{j}=\Phi_{\delta_{j}}=\left(-\Delta I+\nabla \nabla^{T}\right) \phi_{\delta_{j}}
$$

where $\phi_{\delta}(\mathbf{x})=\delta^{-d} \phi(\mathbf{x} / \delta)$ and $\phi$ is a function satisfying the decay condition (2.2) with $\tau=\sigma+1$ and $\sigma>d / 2$. These kernels generate for any $0<\delta \leqslant \delta_{c}$ native spaces which are norm equivalent to the same Sobolev space which is determined by the smoothness of the kernel.

LEMMA 3.1 Let $\delta \in\left(0, \delta_{c}\right]$. Let $\phi$ generate $H^{\sigma+1}\left(\mathbb{R}^{d}\right)$ with $\sigma>d / 2$. Then

$$
\mathscr{N}_{\Phi_{\delta}}\left(\mathbb{R}^{d}\right)=\widetilde{\mathbf{H}}^{\sigma}\left(\mathbb{R}^{d}, \operatorname{div}\right)
$$


and for every $\mathbf{v} \in \widetilde{\mathbf{H}}^{\sigma}\left(\mathbb{R}^{d}, \operatorname{div}\right)$, we have

$$
C_{1}\|\mathbf{v}\|_{\mathscr{N}_{\Phi_{\delta}}\left(\mathbb{R}^{d}\right)}^{2} \leqslant\|\mathbf{v}\|_{\tilde{\mathbf{H}}^{\sigma}\left(\mathbb{R}^{d}, \text { div }\right)}^{2} \leqslant C_{2} \delta^{-2(\sigma+1)}\|\mathbf{v}\|_{\mathscr{N}_{\Phi_{\delta}}\left(\mathbb{R}^{d}\right)}^{2}
$$

for two positive constants $C_{1}, C_{2}$.

Proof. We first consider the upper bound. If $\delta \leqslant 1$, then

$$
\left(1+\|\boldsymbol{\omega}\|_{2}^{2}\right)^{\sigma+1}=\delta^{-2(\sigma+1)}\left(\delta^{2}+\|\delta \boldsymbol{\omega}\|_{2}^{2}\right)^{\sigma+1} \leqslant \delta^{-2(\sigma+1)}\left(1+\|\delta \boldsymbol{\omega}\|_{2}^{2}\right)^{\sigma+1} .
$$

Moreover, if $1<\delta \leqslant \delta_{c}$, we can simply use

$$
\left(1+\|\boldsymbol{\omega}\|_{2}^{2}\right)^{\sigma+1} \leqslant\left(1+\|\delta \boldsymbol{\omega}\|_{2}^{2}\right)^{\sigma+1} \leqslant \delta^{-2(\sigma+1)} \delta_{c}^{2(\sigma+1)}\left(1+\|\delta \boldsymbol{\omega}\|_{2}^{2}\right)^{\sigma+1} .
$$

With both estimates we can then deduce for $m=\max \left\{1, \delta_{c}^{\sigma+1}\right\}$

$$
\begin{aligned}
& \|\mathbf{v}\|_{\widetilde{\mathbf{H}}^{\sigma}\left(\mathbb{R}^{d}, \text { div }\right)}^{2}=(2 \pi)^{-d / 2} \int_{\mathbb{R}^{d}} \frac{\|\widehat{\mathbf{v}}(\boldsymbol{\omega})\|_{2}^{2}}{\|\boldsymbol{\omega}\|_{2}^{2}}\left(1+\|\boldsymbol{\omega}\|_{2}^{2}\right)^{\sigma+1} d \boldsymbol{\omega} \\
& \leqslant \frac{m^{2}}{(2 \pi)^{d / 2}} \int_{\mathbb{R}^{d}} \frac{\|\widehat{\mathbf{v}}(\boldsymbol{\omega})\|_{2}^{2}}{\delta^{2(\sigma+1)}\|\boldsymbol{\omega}\|_{2}^{2}}\left(1+\|\delta \boldsymbol{\omega}\|_{2}^{2}\right)^{\sigma+1} d \boldsymbol{\omega} \\
& \leqslant m^{2} c_{2}(2 \pi)^{-d / 2} \int_{\mathbb{R}^{d}} \delta^{-2(\sigma+1)} \frac{\|\widehat{\mathbf{v}}(\boldsymbol{\omega})\|_{2}^{2}}{\|\boldsymbol{\omega}\|_{2}^{2} \widehat{\boldsymbol{\phi}}(\delta \boldsymbol{\omega})} d \boldsymbol{\omega} \\
& =m^{2} c_{2}(2 \pi)^{-d / 2} \int_{\mathbb{R}^{d}} \delta^{-2(\sigma+1)} \frac{\|\boldsymbol{\mathbf { v }}(\boldsymbol{\omega})\|_{2}^{2}}{\|\boldsymbol{\omega}\|_{2}^{2} \widehat{\phi}_{\delta}(\boldsymbol{\omega})} d \boldsymbol{\omega} \\
& =C_{2} \delta^{-2(\sigma+1)}\|\mathbf{v}\|_{\mathcal{N}_{\Phi_{\delta}}}^{2}\left(\mathbb{R}^{d}\right)
\end{aligned}
$$

where $C_{2}=m^{2} c_{2}$. For the lower bound, again, we distinguish between two cases. If $\delta \leqslant 1$, then we have

$$
\left(1+\|\boldsymbol{\omega}\|_{2}^{2}\right)^{\sigma+1} \geqslant\left(1+\|\delta \boldsymbol{\omega}\|_{2}^{2}\right)^{\sigma+1} .
$$

Moreover, for $1<\delta \leqslant \delta_{c}$, we have

$$
\left(1+\|\boldsymbol{\omega}\|_{2}^{2}\right)^{\sigma+1}=\delta^{-2(\sigma+1)}\left(\delta^{2}+\|\delta \boldsymbol{\omega}\|_{2}^{2}\right)^{\sigma+1} \geqslant \delta_{c}^{-2(\sigma+1)}\left(1+\|\delta \boldsymbol{\omega}\|_{2}^{2}\right)^{\sigma+1} .
$$

Similarly, for $k=\min \left\{1, \delta_{c}^{-\sigma-1}\right\}$, we then find

$$
\begin{aligned}
& \|\mathbf{v}\|_{\widetilde{\mathbf{H}}^{\sigma}\left(\mathbb{R}^{d}, \mathrm{div}\right)}^{2} \geqslant k^{2}(2 \pi)^{-d / 2} \int_{\mathbb{R}^{d}} \frac{\|\widehat{\mathbf{v}}(\boldsymbol{\omega})\|_{2}^{2}}{\|\boldsymbol{\omega}\|_{2}^{2}}\left(1+\|\delta \boldsymbol{\omega}\|_{2}^{2}\right)^{\sigma+1} d \boldsymbol{\omega} \\
& \geqslant k^{2} c_{1}(2 \pi)^{-d / 2} \int_{\mathbb{R}^{d}} \frac{\|\widehat{\mathbf{v}}(\boldsymbol{\omega})\|_{2}^{2}}{\|\boldsymbol{\omega}\|_{2}^{2} \widehat{\phi_{\delta}}(\boldsymbol{\omega})} d \boldsymbol{\omega} \\
& =C_{1}\|\mathbf{v}\|_{\mathscr{N}_{\Phi_{\delta}}\left(\mathbb{R}^{d}\right)}^{2},
\end{aligned}
$$

where $C_{1}=k^{2} c_{1}$.

Another ingredient of the multilevel convergence proof consists of a sampling inequality for vectorvalued functions. This result comes from and is proven in Wendland (2009). It gives an error estimate in terms of the fill distance or mesh norm of a discrete point set $X \subseteq \Omega$ defined by

$$
h_{X, \Omega}=\sup _{\mathbf{x} \in \Omega} \inf _{\mathbf{x}_{j} \in X}\left\|\mathbf{x}-\mathbf{x}_{j}\right\|_{2} .
$$


LEMMA 3.2 Let $\Omega \subseteq \mathbb{R}^{d}$ be a domain with Lipschitz boundary. Furthermore, let $\sigma, \eta \in \mathbb{R}$ with $\sigma>d / 2$ and $0 \leqslant \eta \leqslant \sigma$. Assume that $\mathbf{u} \in \mathbf{H}^{\sigma}(\Omega)$ satisfies $\left.\mathbf{u}\right|_{X}=\mathbf{0}$ on a discrete set $X \subseteq \Omega$ with a sufficiently small fill distance $h_{X, \Omega}$. Then,

$$
\|\mathbf{u}\|_{\mathbf{H}^{\eta}(\Omega)} \leqslant C h_{X, \Omega}^{\sigma-\eta}\|\mathbf{u}\|_{\mathbf{H}^{\sigma}(\Omega)}
$$

with a constant $C>0$ independent of $\mathbf{u}$.

\subsection{The Multilevel Interpolation Algorithm}

Suppose we are given a sequence of point sets in $\Omega$, namely $X_{1}, X_{2}, \ldots$, with decreasing mesh norms $h_{1}:=h_{X_{1}, \Omega}, h_{2}:=h_{X_{2}, \Omega}, \ldots$. These point sets do not need to be nested. However, if they are not nested the algorithm below will create an interpolant only for the finest point set.

From these mesh norms we construct support radii so that

$$
\delta_{j}=v h_{j}^{\frac{\sigma}{\sigma+1}}
$$

with some proportionality constant $v>0$. Equality in (3.2) requires the knowledge of the fill distances $h_{j}$. These can be computed by discretising the domain $\Omega$ and replacing the supremum in the definition $h_{j}$ by a maximum over the discretised set. To further speed up this process the discretised set can be replaced by the data set itself, i.e. we approximate $h_{X, \Omega}$ by $\max _{x \in X} \min _{y \in X \backslash\{x\}}\|x-y\|_{2}$. Using a data structure for nearest neighbour searching allows us to compute this number in $\mathscr{O}(N \log N)$ time.

Furthermore, in practical applications often only one data set $X$ is given and the sequence of then nested data sets $X_{1} \subseteq X_{2} \subseteq \cdots \subseteq X_{k}=X$ has to be constructed using, for example, thinning algorithms like those in Floater \& Iske (1998).

We define scaled kernels as in (3.1). The vector-valued divergence-free interpolants $\mathbf{s}_{j}$ come from the space

$$
\mathbf{V}_{j}=\left\{\sum_{\mathbf{x} \in X_{j}} \Phi_{j}(\cdot-\mathbf{x}) \boldsymbol{\beta}_{\mathbf{x}} \mid \boldsymbol{\beta}_{\mathbf{x}} \in \mathbb{R}^{d}\right\}
$$

Now, we can state the multilevel interpolation algorithm for matrix-valued kernels, which reconstructs some divergence-free target function $\mathbf{u}$ by a residual correction scheme.

Algorithm 3.1 (Multilevel Interpolation Algorithm)

Input: Data $\mathbf{u}$ with $\operatorname{div}(\mathbf{u})=0$ and number of levels $n$

Set $\mathbf{u}_{0}=\mathbf{0}$ and $\mathbf{e}_{0}=\mathbf{u}$. For $j=1, \ldots, n$ do

(i) Determine the local correction $\mathbf{s}_{j} \in \mathbf{V}_{j}$ to $\mathbf{e}_{j-1}$ on $X_{j}$

$$
\mathbf{s}_{j}(\mathbf{x})=\mathbf{e}_{j-1}(\mathbf{x}) \quad \mathbf{x} \in X_{j}
$$

(ii) Update the global approximation and the residuals

$$
\begin{aligned}
\mathbf{u}_{j} & =\mathbf{u}_{j-1}+\mathbf{s}_{j} \\
\mathbf{e}_{j} & =\mathbf{e}_{j-1}-\mathbf{s}_{j}
\end{aligned}
$$

Output: Approximate solution $\mathbf{u}_{n}$ to $\mathbf{u}$ 
Note that we only need to know the unknown function $\mathbf{u}$ at the discrete data sites $X_{j}$.

The residual in this case is in fact the error between the solution $\mathbf{u}$ and its $j$ th multilevel approximation $\mathbf{u}_{j}$, i.e.

$$
\mathbf{e}_{j}=\mathbf{u}-\mathbf{u}_{j} .
$$

Our main result of this paper shows that the above algorithm indeed converges under the assumption (3.2) on the relation between the support radii $\delta_{j}$ and the fill distances $h_{j}$.

THEOREM 3.2 Let $\sigma>d / 2$ with $d=2,3$. Let $\Omega \subseteq \mathbb{R}^{d}$ be bounded with a $C^{k, 1}$ boundary where $k \geqslant \sigma$. Let $X_{1}, X_{2}, \ldots$ be a sequence of point sets in $\Omega$ with mesh norms $h_{1}=h_{X_{1}, \Omega}, h_{2}=h_{X_{2}, \Omega}, \ldots$. Assume

$$
\gamma \mu h_{j} \leqslant h_{j+1} \leqslant \mu h_{j}
$$

for $j=1,2, \ldots$ and some fixed $\mu \in(0,1)$ and $\gamma \in(0,1)$. Suppose that $\phi \in L_{1}\left(\mathbb{R}^{d}\right)$ satisfies (2.2) with $\tau=\sigma+1$, i.e. that its native space is $H^{\sigma+1}\left(\mathbb{R}^{d}\right)$. Define

$$
\delta_{j}=\left(\frac{h_{j}}{\mu}\right)^{\frac{\sigma}{\sigma+1}} \quad \text { as well as } \quad \Phi_{j}=\Phi_{\delta_{j}}=\left(-\Delta I+\nabla \nabla^{T}\right) \phi_{\delta_{j}}
$$

with $\phi_{\delta}=\delta^{-d} \phi(\cdot / \delta)$. Let $h_{1} \leqslant \mu$ be sufficiently small. Finally, let $\mathbf{u} \in \mathbf{H}^{\sigma}(\Omega$, div $)$. Then, there exists a positive constant $C$ independent of $\mu, j$ and $\mathbf{u}$ such that

$$
\left\|\mathbf{E}_{\mathrm{div}} \mathbf{e}_{j}\right\|_{\Phi_{j+1}} \leqslant \alpha\left\|\mathbf{E}_{\mathrm{div}} \mathbf{e}_{j-1}\right\|_{\Phi_{j}}
$$

for $j=1,2, \ldots$ with $\alpha=C \mu^{\sigma}$. Moreover, we have the estimates

$$
\left\|\mathbf{u}-\mathbf{u}_{n}\right\|_{\mathbf{L}_{2}(\Omega)} \leqslant \widetilde{C} \alpha^{n}\|\mathbf{u}\|_{\mathbf{H}^{\sigma}(\Omega)}
$$

for $n=1,2, \ldots$ with another constant $\widetilde{C}>0$ independent of $n, \alpha$ and $\mathbf{u}$. Hence, the multilevel approximation $\mathbf{u}_{n}$ converges to $\mathbf{u}$ in the $L_{2}$ norm if we choose $\mu$ so small that $\alpha<1$.

Proof. It is easy to verify that the support radii are monotonically decreasing. We compute

$$
\begin{aligned}
& \left\|\mathbf{E}_{\mathrm{div}} \mathbf{e}_{j}\right\|_{\Phi_{j+1}}^{2}=(2 \pi)^{-d / 2} \int_{\mathbb{R}^{d}} \frac{\left\|\widehat{\mathbf{E}_{\mathrm{div}} \mathbf{e}_{j}}(\boldsymbol{\omega})\right\|_{2}^{2}}{\|\boldsymbol{\omega}\|_{2}^{2} \widehat{\boldsymbol{\phi}_{j+1}}(\boldsymbol{\omega})} d \boldsymbol{\omega} \\
& \leqslant \frac{1}{c_{1}}(2 \pi)^{-d / 2} \int_{\mathbb{R}^{d}} \frac{\left\|\widehat{\mathbf{E}_{\mathrm{div}} \mathbf{e}_{j}}(\boldsymbol{\omega})\right\|_{2}^{2}}{\|\boldsymbol{\omega}\|_{2}^{2}}\left(1+\left\|\delta_{j+1} \boldsymbol{\omega}\right\|_{2}^{2}\right)^{\sigma+1} d \boldsymbol{\omega} \\
& =: \frac{1}{c_{1}}(2 \pi)^{-d / 2}\left(I_{1}+I_{2}\right) \text {, }
\end{aligned}
$$

where we introduced the notation

$$
\begin{aligned}
I_{1} & :=\int_{\|\boldsymbol{\omega}\|_{2} \leqslant \frac{1}{\delta_{j+1}}} \frac{\left\|\widehat{\mathbf{E}_{\operatorname{div}} \mathbf{e}_{j}}(\boldsymbol{\omega})\right\|_{2}^{2}}{\|\boldsymbol{\omega}\|_{2}^{2}}\left(1+\left\|\delta_{j+1} \boldsymbol{\omega}\right\|_{2}^{2}\right)^{\sigma+1} d \boldsymbol{\omega}, \\
I_{2}:= & \int_{\|\boldsymbol{\omega}\|_{2} \geqslant \frac{1}{\delta_{j+1}}} \frac{\left\|\widehat{\mathbf{E}_{\operatorname{div}} \mathbf{e}_{j}}(\boldsymbol{\omega})\right\|_{2}^{2}}{\|\boldsymbol{\omega}\|_{2}^{2}}\left(1+\left\|\delta_{j+1} \boldsymbol{\omega}\right\|_{2}^{2}\right)^{\sigma+1} d \boldsymbol{\omega} .
\end{aligned}
$$


To bound these two integrals we start with the following observation:

$$
\begin{aligned}
\left\|\mathbf{e}_{j}\right\|_{\mathbf{H}^{\sigma}(\Omega)}^{2} & =\left\|\mathbf{e}_{j-1}-\mathbf{s}_{\mathbf{e}_{j-1}}\right\|_{\mathbf{H}^{\sigma}(\Omega)}^{2} \\
& =\left\|\mathbf{E}_{\mathrm{div}} \mathbf{e}_{j-1}-\mathbf{s}_{\mathbf{E}_{\mathrm{div}} \mathbf{e}_{j-1}}\right\|_{\mathbf{H}^{\sigma}(\Omega)}^{2} \\
& \leqslant\left\|\mathbf{E}_{\mathrm{div}} \mathbf{e}_{j-1}-\mathbf{s}_{\mathbf{E}_{\mathrm{div}} \mathbf{e}_{j-1}}\right\|_{\mathbf{H}^{\sigma}\left(\mathbb{R}^{d}\right)}^{2} \\
& \leqslant\left\|\mathbf{E}_{\mathrm{div}} \mathbf{e}_{j-1}-\mathbf{s}_{\mathbf{E}_{\mathrm{div}} \mathbf{e}_{j-1}}\right\|_{\widetilde{\mathbf{H}}^{\sigma}\left(\mathbb{R}^{d}\right)}^{2} \\
& \leqslant C \delta_{j}^{-2(\sigma+1)}\left\|\mathbf{E}_{\mathrm{div}} \mathbf{e}_{j-1}-\mathbf{s}_{\mathbf{E}_{\mathrm{div}} \mathbf{e}_{j-1}}\right\|_{\Phi_{j}}^{2} \\
& \leqslant C \delta_{j}^{-2(\sigma+1)}\left\|\mathbf{E}_{\mathrm{div}} \mathbf{e}_{j-1}\right\|_{\Phi_{j}}^{2} .
\end{aligned}
$$

Here we have used the definition of $\mathbf{e}_{j}$, the facts that $\mathbf{E}_{\mathrm{div}} \mathbf{e}_{j-1}=\mathbf{e}_{j-1}$ on $\Omega$ and that the interpolant $\mathbf{s}_{\mathbf{e}_{j-1}}$ equals $\mathbf{S}_{\mathbf{E}_{\mathrm{div}} \mathbf{e}_{j-1}}$ as well as (2.5), Lemma 3.1 and the best approximation property of the interpolant.

For the first integral, $I_{1}$, we have $\delta_{j+1}\|\boldsymbol{\omega}\|_{2} \leqslant 1$ and thus by Lemma 2.1, the sampling inequality (Lemma 3.2) and (3.5), it follows that

$$
\begin{aligned}
I_{1} & \leqslant 2^{\sigma+1} \int_{\|\boldsymbol{\omega}\|_{2} \leqslant \frac{1}{\delta_{j+1}}} \frac{\left\|\widehat{\mathbf{E}_{\mathrm{div}} \mathbf{e}_{j}}(\boldsymbol{\omega})\right\|_{2}^{2}}{\|\boldsymbol{\omega}\|_{2}^{2}} d \boldsymbol{\omega} \\
& \leqslant C\left\|\mathbf{e}_{j}\right\|_{\mathbf{L}_{2}(\Omega)}^{2} \\
& \leqslant C h_{j}^{2 \sigma}\left\|\mathbf{e}_{j}\right\|_{\mathbf{H}^{\sigma}(\Omega)}^{2} \\
& \leqslant C h_{j}^{2 \sigma} \delta_{j}^{-2(\sigma+1)}\left\|\mathbf{E}_{\mathrm{div}} \mathbf{e}_{j-1}\right\|_{\Phi_{j}}^{2} \\
& =C_{1} \mu^{2 \sigma}\left\|\mathbf{E}_{\mathrm{div}} \mathbf{e}_{j-1}\right\|_{\Phi_{j}}^{2},
\end{aligned}
$$

using also that by definition we have

$$
\frac{h_{j}^{\sigma}}{\delta_{j}^{\sigma+1}}=\mu^{\sigma} .
$$

Next we turn to the second integral $I_{2}$. Since $\delta_{j+1}\|\boldsymbol{\omega}\|_{2} \geqslant 1$, we find

$$
\left(1+\delta_{j+1}^{2}\|\boldsymbol{\omega}\|_{2}^{2}\right)^{\sigma+1} \leqslant\left(2 \delta_{j+1}^{2}\|\boldsymbol{\omega}\|_{2}^{2}\right)^{\sigma+1} \leqslant 2^{\sigma+1} \delta_{j+1}^{2(\sigma+1)}\left(1+\|\boldsymbol{\omega}\|_{2}^{2}\right)^{\sigma+1}
$$

and consequently, using Proposition 2.5 and (3.5) once again, gives

$$
\begin{aligned}
I_{2} & \leqslant 2^{\sigma+1} \delta_{j+1}^{2(\sigma+1)} \int_{\mathbb{R}^{d}} \frac{\left\|\mathbf{E}_{\mathrm{div}} \mathbf{e}_{j}(\boldsymbol{\omega})\right\|_{2}^{2}}{\|\boldsymbol{\omega}\|_{2}^{2}}\left(1+\|\boldsymbol{\omega}\|_{2}^{2}\right)^{\sigma+1} d \boldsymbol{\omega} \\
& =2^{\sigma+1} \delta_{j+1}^{2(\sigma+1)}\left\|\mathbf{E}_{\mathrm{div}} \mathbf{e}_{j}\right\|_{\tilde{\mathbf{H}}^{\sigma}\left(\mathbb{R}^{d}, \text { div }\right)}^{2} \\
& \leqslant C \delta_{j+1}^{2(\sigma+1)}\left\|\mathbf{e}_{j}\right\|_{\mathbf{H}^{\sigma}(\Omega)}^{2} \\
& \leqslant C\left(\delta_{j+1} / \delta_{j}\right)^{2(\sigma+1)}\left\|\mathbf{E}_{\mathrm{div}} \mathbf{e}_{j-1}\right\|_{\Phi_{j}}^{2} \\
& \leqslant C_{2} \mu^{2 \sigma}\left\|\mathbf{E}_{\mathrm{div}} \mathbf{e}_{j-1}\right\|_{\Phi_{j}}^{2} .
\end{aligned}
$$

In the last step we have used

$$
\left(\frac{\delta_{j+1}}{\delta_{j}}\right)^{\sigma+1}=\left(\frac{h_{j+1}}{\mu}\right)^{\sigma}\left(\frac{\mu}{h_{j}}\right)^{\sigma}=\left(\frac{h_{j+1}}{h_{j}}\right)^{\sigma} \leqslant \mu^{\sigma} .
$$


Combining both estimates now yields (3.4) with

$$
\alpha=\sqrt{\frac{1}{c_{1}}(2 \pi)^{-d / 2}}\left(C_{1}+C_{2}\right)^{1 / 2} \mu^{\sigma}=C \mu^{\sigma} .
$$

It follows by the sampling inequality, (2.5) and Lemma 3.1 that

$$
\begin{aligned}
\left\|\mathbf{u}-\mathbf{u}_{n}\right\|_{\mathbf{L}_{2}(\Omega)}^{2} & =\left\|\mathbf{e}_{n}\right\|_{\mathbf{L}_{2}(\Omega)}^{2} \leqslant C h_{n}^{2 \sigma}\left\|\mathbf{e}_{n}\right\|_{\mathbf{H}^{\sigma}(\Omega)}^{2} \\
& \leqslant C h_{n}^{2 \sigma}\left\|\mathbf{E}_{\mathrm{div}} \mathbf{e}_{n}\right\|_{\widetilde{\mathbf{H}}^{\sigma}\left(\mathbb{R}^{d}\right)} \\
& \leqslant C h_{n}^{2 \sigma} \delta_{n+1}^{-2(\sigma+1)}\left\|\mathbf{E}_{\mathrm{div}} \mathbf{e}_{n}\right\|_{\Phi_{n+1}}^{2} \\
& =C\left\|\mathbf{E}_{\mathrm{div}} \mathbf{e}_{n}\right\|_{\Phi_{n+1}}^{2}
\end{aligned}
$$

where we have used the fact that

$$
\frac{h_{n}^{\sigma}}{\delta_{n+1}^{\sigma+1}}=\mu^{\sigma}\left(\frac{h_{n}}{h_{n+1}}\right)^{\sigma} \leqslant \mu^{\sigma}\left(\frac{1}{\mu \gamma}\right)^{\sigma}=\gamma^{-\sigma} .
$$

Applying (3.4) $n$ times we can conclude that

$$
\begin{aligned}
\left\|\mathbf{u}-\mathbf{u}_{n}\right\|_{\mathbf{L}_{2}(\Omega)}^{2} & \leqslant \widetilde{C} \alpha^{2 n}\left\|\mathbf{E}_{\mathrm{div}} \mathbf{e}_{0}\right\|_{\Phi_{1}}^{2}=\widetilde{C} \alpha^{2 n}\left\|\mathbf{E}_{\mathrm{div}} \mathbf{u}\right\|_{\Phi_{1}}^{2} \\
& \leqslant \widetilde{C} \alpha^{2 n}\|\mathbf{u}\|_{\mathbf{H}^{\sigma}(\Omega)}^{2}
\end{aligned}
$$

with a constant $\widetilde{C}=\widetilde{C}(\gamma)>0$ independent of $\alpha, k$ and $\mathbf{u}$.

In contrast to the multilevel interpolation with scalar-valued kernels as in Wendland (2010), the multilevel interpolation of divergence-free fields with matrix-valued kernels is shown to converge only for a (mildly) non-proportional relationship between mesh norm and support radius. In fact, as we will see in the last section, a proportional relationship does not lead to convergence. The non-proportionality is introduced via the native space norm for matrix-valued kernels.

It is even possible to derive convergence results for the derivatives of the error and predict convergence orders in terms of the fill distance of the finest grid. To see this, we will assume that the fill distances from level to level shrink according to $h_{j+1}=\mu h_{j}$, which is a slightly stronger assumption than the one in (3.3), since we give up the leeway introduced by the constant $\gamma$ and now require a uniform refinement of the fill distances from level to level.

THEOREM 3.3 Let $\varepsilon>0$ and $0 \leqslant \beta \leqslant \sigma-\varepsilon$. Let the assumptions of Theorem 3.2 with (3.3) replaced by $h_{j+1}=\mu h_{j}$ hold. Then, if $\mu$ is chosen sufficiently small and if $h_{1}=\mu$ we have the error estimate

$$
\left\|\mathbf{u}-\mathbf{u}_{n}\right\|_{\mathbf{H}^{\beta}(\Omega)} \leqslant \widetilde{C} h_{n}^{\sigma-\beta-\varepsilon}\|\mathbf{u}\|_{\mathbf{H}^{\sigma}(\Omega)}
$$

for $n=1,2, \ldots$ with a constant $\widetilde{C}>0$ independent of $n, \beta, \varepsilon$ and $\mathbf{u}$.

Proof. From the proof of Theorem 3.2 we know that the error between two levels can be bounded by

$$
\left\|\mathbf{E}_{\mathrm{div}} \mathbf{e}_{j}\right\|_{\Phi_{j+1}} \leqslant \alpha\left\|\mathbf{E}_{\mathrm{div}} \mathbf{e}_{j-1}\right\|_{\Phi_{j}}
$$

with $\alpha=C \mu^{\sigma}$ where $C$ is independent of the level. If we now choose $\mu \in(0,1)$ so small that $C \mu^{\varepsilon}<1$, then (3.6) becomes

$$
\left\|\mathbf{E}_{\mathrm{div}} \mathbf{e}_{j}\right\|_{\Phi_{j+1}} \leqslant \mu^{\sigma-\varepsilon}\left\|\mathbf{E}_{\mathrm{div}} \mathbf{e}_{j-1}\right\|_{\Phi_{j}}
$$


which leads iteratively for the finest level, as in the proof of Theorem 3.2, to

$$
\left\|\mathbf{E}_{\mathrm{div}} \mathbf{e}_{n}\right\|_{\Phi_{n+1}} \leqslant \mu^{(\sigma-\varepsilon) n}\|\mathbf{u}\|_{\mathbf{H}^{\sigma}(\Omega)} .
$$

From $h_{j+1}=\mu h_{j}$ it follows that $h_{n}=\mu^{n-1} h_{1}=\mu^{n}$ which then gives

$$
\left\|\mathbf{E}_{\mathrm{div}} \mathbf{e}_{n}\right\|_{\Phi_{n+1}} \leqslant h_{n}^{\sigma-\varepsilon}\|\mathbf{u}\|_{\mathbf{H}^{\sigma}(\Omega)} .
$$

To derive the stated inequality, we proceed as before. Using the sampling inequality, (2.5) and Lemma 3.1 we see that

$$
\begin{aligned}
\left\|\mathbf{u}-\mathbf{u}_{n}\right\|_{\mathbf{H}^{\beta}(\Omega)} & =\left\|\mathbf{e}_{n}\right\|_{\mathbf{H}^{\beta}(\Omega)} \leqslant C h_{n}^{\sigma-\beta}\left\|\mathbf{e}_{n}\right\|_{\mathbf{H}^{\sigma}(\Omega)} \\
& \leqslant C h_{n}^{\sigma-\beta}\left\|\mathbf{E}_{\operatorname{div}} \mathbf{e}_{n}\right\|_{\widetilde{\mathbf{H}}^{\sigma}\left(\mathbb{R}^{d}\right)} \\
& \leqslant C h_{n}^{\sigma-\beta} \delta_{n+1}^{-(\sigma+1)}\left\|\mathbf{E}_{\mathrm{div}} \mathbf{e}_{n}\right\|_{\Phi_{n+1}} \\
& =C h_{n}^{-\beta}\left\|\mathbf{E}_{\mathrm{div}} \mathbf{e}_{n}\right\|_{\Phi_{n+1}},
\end{aligned}
$$

which gives together with (3.7) the desired bound.

Since we can choose $\varepsilon>0$ arbitrarily small we can come as close as we want to the optimal order $\sigma-\beta$. However, the price for this is that we have to choose $\mu$ correspondingly small.

\subsection{Stability}

In this section, we will discuss how the interpolation matrices $A_{\Phi_{\delta}, X}$ in the multilevel algorithm depend on the support radii and the separation distance

$$
q_{X}:=\min _{j \neq k}\left\|\mathbf{x}_{j}-\mathbf{x}_{k}\right\|_{2}
$$

for some data set $X=\left\{\mathbf{x}_{1}, \ldots, \mathbf{x}_{N}\right\}$. We will extend the one-shot results proven by Fuselier (Fuselier (2008b,a)). Earlier work is due to Lowitzsch (2005c); Narcowich \& Ward (1994). Since $A_{\Phi_{\delta}, X}$ is symmetric and positive definite, we only need to find bounds on the smallest and largest eigenvalues.

For the smallest eigenvalue we will use the following general result by Fuselier (Fuselier (2008b,a)).

THEOREM 3.4 Let $\phi$ be an even positive definite function, which possesses a positive Fourier transform $\widehat{\phi} \in C\left(\mathbb{R}^{d} /\{\boldsymbol{0}\}\right)$. Let $\Phi=\left(-\Delta I+\nabla \nabla^{T}\right) \phi$. Define the function

$$
M(s):=\inf _{\|\boldsymbol{\omega}\|_{2} \leqslant s} \widehat{\phi}(\boldsymbol{\omega}) .
$$

A lower bound on the smallest eigenvalue of the interpolation matrix $A_{\Phi, X}$ is given by

$$
\lambda_{\min }\left(A_{\Phi, X}\right) \geqslant\left(\frac{s^{2}}{16 \pi}\right)^{(d+2) / 2} \frac{M(s) \pi}{(4 \pi)^{d} \Gamma((d+2) / 2)}
$$

for any $s>0$ satisfying

$$
s \geqslant \widetilde{c} / q_{X}
$$

where $\widetilde{c}$ is a constant independent of $\phi$ and $X$. 
A direct consequence of this result is the following one. Suppose $\phi \in L_{1}\left(\mathbb{R}^{d}\right)$ has a Fourier transform satisfying the bound (2.2) with $\tau=\sigma+1$, meaning in particular $\widehat{\phi}(\boldsymbol{\omega}) \geqslant c_{1}\left(1+\|\boldsymbol{\omega}\|_{2}^{2}\right)^{-\sigma-1}$. Then, obviously, $M(s)$ can be bounded from below by $M(s) \geqslant c_{1}\left(1+s^{2}\right)^{-\sigma-1} \geqslant c s^{-2 \sigma-2}$, where the last estimate holds for example for $s \geqslant 1$. Hence, taking $s=c / q_{X}$, Theorem 3.4 yields in this situation,

$$
\lambda_{\min }\left(A_{\Phi, X}\right) \geqslant c q_{X}^{-(d+2)-2 \sigma-2}=c q_{X}^{2 \sigma-d} .
$$

Here, however, we are interested in scaled versions of the basis function, i. e. we are interested in lower bounds for $\lambda_{\min }\left(A_{\Phi_{\delta}, X}\right)$ with $\Phi_{\delta}=\left(-\Delta I+\nabla \nabla^{T}\right) \phi_{\delta}$, where $\phi_{\delta}=\delta^{-d} \phi(\cdot / \delta)$. We can derive such lower bounds in two ways. We can either proceed as above using the fact that $\widehat{\phi_{\delta}}=\widehat{\phi}(\delta \cdot)$ and hence $M\left(c / q_{X}\right) \geqslant c\left(q_{X} / \delta\right)^{2(\sigma+1)}$ yielding

$$
\lambda_{\min }\left(A_{\Phi_{\delta}, X}\right) \geqslant c q_{X}^{-(d+2)}\left(\frac{q_{X}}{\delta}\right)^{2(\sigma+1)}=c q_{X}^{2 \sigma-d} \delta^{-2 \sigma-2}
$$

for $\delta / q_{X} \geqslant 1$. Alternatively, we can realise that

$$
\Phi_{\delta}=\delta^{-d-2}\left[\left(-\Delta I+\nabla \nabla^{T}\right) \phi\right](\cdot / \delta)=\delta^{-d-2} \Phi(\cdot / \delta)
$$

and that $X / \delta:=\{\mathbf{x} / \delta \mid \mathbf{x} \in X\}$ has obviously separation distance $q_{X / \delta}=q_{X} / \delta$. Hence, apart from the scaling factor $\delta^{-d-2}$ interpolating with $\Phi_{\delta}$ in $X$ is the same as interpolating with $\Phi$ in $X / \delta$. Thus, (3.8) yields

$$
\lambda_{\min }\left(A_{\Phi_{\delta}, X}\right) \geqslant c \delta^{-d-2}\left(\frac{q_{X}}{\delta}\right)^{2 \sigma-d}=c q_{X}^{2 \sigma-d} \delta^{-2 \sigma-2}
$$

which is obviously the same as (3.9).

THEOREM 3.5 Suppose $\phi$ is positive definite and compactly supported, with a Fourier transform satisfying (2.2) with $\tau=\sigma+1$ and $\sigma>d / 2$, i. e. generating $H^{\sigma+1}\left(\mathbb{R}^{d}\right)$. Then,

$$
\operatorname{cond}\left(A_{\Phi_{\delta}, X}\right) \leqslant C\left(1+\frac{2 \delta}{q_{X}}\right)^{d}\left(\frac{\delta}{q_{X}}\right)^{2 \sigma-d}
$$

with a constant $C>0$ independent of $q_{X}$ and $\delta$.

Proof. It remains to bound the largest eigenvalue $\lambda_{\max }=\lambda_{\max }\left(A_{\Phi_{\delta}, X}\right)$. For this, we follow ideas from Farrell \& Wendland (2013). Due to the Gershgorin theorem there is an index $j \in\{1, \ldots, d N\}$ with corresponding data site $\mathbf{x}_{\tilde{j}}$ with $1 \leqslant \widetilde{j} \leqslant N$ such that

$$
\left|\lambda_{\max }\right| \leqslant \sum_{k=1}^{d N}\left|a_{j k}^{\delta}\right|
$$

with $A_{\Phi_{\delta}, X}=\left(a_{j k}^{\delta}\right) \in \mathbb{R}^{d N \times d N}$. The entries $a_{j k}^{\delta}$ have the form

$$
a_{j k}^{\delta}=\delta^{-d-2}\left(D^{\alpha} \phi\right)\left(\left(\mathbf{x}_{\tilde{j}}-\mathbf{x}_{\tilde{k}}\right) / \delta\right)
$$

with certain multi-indices $\alpha \in \mathbb{N}_{0}^{d}$ of length $|\alpha|=2$. Note that the data site $\mathbf{x}_{\tilde{k}}$ corresponds to $d$ indices $k$. By assumption, $\phi$ belongs to $C^{2}\left(\mathbb{R}^{d}\right)$ and has compact support. This means that there is a constant $c_{\phi}>0$ such that

$$
\left|a_{j k}^{\delta}\right| \leqslant \delta^{-d-2} c_{\phi}, \quad 1 \leqslant j, k \leqslant d N
$$


Since $\Phi_{\delta}$ has compact support in the ball $B(\mathbf{0}, \delta)$, only those terms $a_{j k}^{\delta}, 1 \leqslant k \leqslant d N$, are nonzero which belong to the index set

$$
I_{\tilde{j}}:=\left\{\widetilde{k} \in\{1, \ldots, N\} \mid\left\|\mathbf{x}_{\tilde{j}}-\mathbf{x}_{\tilde{k}}\right\|_{2}<\delta\right\}
$$

That is, there are $d n_{\tilde{j}}$ non-zero summands with

$$
n_{\tilde{j}}:=\left|\left\{\widetilde{k} \in\{1, \ldots, N\} \mid\left\|\mathbf{x}_{\tilde{j}}-\mathbf{x}_{\tilde{k}}\right\|_{2}<\delta\right\}\right|
$$

In Farrell \& Wendland (2013) it is shown that we have the estimate

$$
n_{\tilde{j}} \leqslant\left(\frac{\delta+q_{X} / 2}{q_{X} / 2}\right)^{2}=\left(1+\frac{2 \delta}{q_{X}}\right)^{d} .
$$

Now we can bound the largest eigenvalue by

$$
\left|\lambda_{\max }\right| \leqslant \sum_{k=1}^{d N}\left|a_{j k}^{\delta}\right| \leqslant d n_{\widetilde{j}_{1 \leqslant k \leqslant N}} \max _{1 \leqslant k}\left|a_{j k}^{\delta}\right| \leqslant c_{\phi} d \delta^{-d-2}\left(1+\frac{2 \delta}{q_{X}}\right)^{d} .
$$

Combining this with the lower bound (3.9) on the smallest eigenvalue yields

$$
\operatorname{cond}\left(A_{\Phi_{\delta}, X}\right) \leqslant C\left(1+\frac{2 \delta}{q_{X}}\right)^{d}\left(\frac{\delta}{q_{X}}\right)^{2 \sigma-d}
$$

with a constant $C>0$ independent of $X$ and $\delta$.

Since we can assume that $2 \delta \geqslant q_{X}$, the bound further simplifies to

$$
\operatorname{cond}\left(A_{\Phi_{\delta}, X}\right) \leqslant C\left(\frac{\delta}{q_{X}}\right)^{2 \sigma}
$$

COROLlaRY 3.1 Under the assumption $\delta=c q_{X}$, the condition number is constant and independent of $q_{X}$ and $\delta$. If we assume that the data sets are quasi-uniform, i.e. satisfy $q_{X} \approx h_{X}$, the convergence theorem requires $\delta=c q_{X}^{\sigma /(\sigma+1)}$. In this case, the condition number behaves like

$$
\operatorname{cond}\left(A_{\Phi_{\delta}, X}\right) \leqslant C q_{X}^{-2 \sigma /(\sigma+1)}
$$

Proof. The statement for the case $\delta=c q_{X}$ immediately follows from (3.10). Since $\delta=c q_{X}^{\sigma /(\sigma+1)}$ gives

$$
\left(\frac{\delta}{q_{X}}\right)^{\sigma}=c \delta^{-1}=c q_{X}^{-\sigma /(\sigma+1)}
$$

the second statement also follows from (3.10). 


\section{Numerical Example}

We will now give a numerical example for our method. The main goal of this example is to verify the theoretical convergence and stability properties outlined in the previous sections. The implementation was done in Matlab using Matlab's inbuilt backslash operator to solve the linear systems. A more sophisticated implementation would use a preconditioned conjugate gradient method.

As a divergence-free vector field we use

$$
\mathbf{u}(x, y)=\left(\begin{array}{c}
-2 x^{3} y \\
3 x^{2} y^{2}
\end{array}\right)
$$

on the closed unit square $\Omega=[0,1]^{2}$. We choose regular nested grids as data sites. That is, $q_{X}$ and $h=h_{X, \Omega}$ are comparable. As basis function we employ $\phi_{2,3}(r)=(1-r)_{+}^{8}\left(32 r^{3}+25 r^{2}+8 r+1\right)$, which generates $H^{\tau}\left(\mathbb{R}^{2}\right)$ with $\tau=\sigma+1=4.5$, see (Wendland, 2005, Theorem 10.35). Furthermore, we use a proportionality constant $v=2.5$ and support radii of the form

$$
\delta=v h^{\frac{\sigma}{\sigma+1}}=v h^{\frac{7}{9}} .
$$

As the numerical results in Tables 1 and 2 show, the numerical solution converges. Note that the number $N$ is actually referring to the amount of data points. The systems that need to be solved are actually twice as big, since $\mathbf{u}$ consists of two spatial components. The parameter $H$ denotes the distance between two horizontal (or vertical) nearest data points of the uniform grid. Thus, this parameter is proportional to the mesh norm $h$. We point out that the condition numbers are estimated values only since the matrices become quite large. The final column shows the ratio of non-zeros to total numbers of entries in the matrix (in percent). The multilevel interpolant and the errors at the last level are depicted in Figures 1 and 2. Due to Corollary 3.1, we expect the condition number to grow asymptotically not faster than $q_{X}^{-1 . \overline{5}}$.

Tables 3 and 4 show the results for the stationary case, i.e.

$$
\delta=v h .
$$

In this case, the algorithm eventually stagnates. According to Corollary 3.1, we expect the condition number to become asymptotically independent of $q_{X}$. The numerics seem to corroborate this.

Finally, Theorem 3.3 also shows us which convergence orders we can expect in our first example and the orders that we see in Tables 1 and 2 are lower than the best possible order. However, in this example, we have chosen $\mu=1 / 2$ which simply might not be small enough for getting close to the optimal order. To improve the order, we have, according to Theorem 3.3, to decrease $\mu$. This, unfortunately, leads to a faster increase in the number of points per level and hence becomes quickly numerically problematic. But the proof of Theorem 3.3 (actually the proof of Theorem 3.2) shows that if we choose $h_{j+1}=\mu h_{j}$ and $\delta_{j}=v h_{j}^{\sigma /(\sigma+1)}$ then the converging factor is determined by two terms:

$$
\begin{aligned}
\left\|\mathbf{E}_{\mathrm{div}} \mathbf{e}_{j}\right\|_{\Phi_{j+1}}^{2} & \leqslant\left[C_{1} \frac{h_{j}^{2 \sigma}}{\delta_{j}^{-2(\sigma+1)}}+C_{2}\left(\frac{\delta_{j+1}}{\delta_{j}}\right)^{2 \sigma}\right]\left\|\mathbf{E}_{\mathrm{div}} \mathbf{e}_{j-1}\right\|_{\Phi_{j}}^{2} \\
& \leqslant\left[C_{1} v^{-2(\sigma+1)}+C_{2} \mu^{2 \sigma}\right]\left\|\mathbf{E}_{\mathrm{div}} \mathbf{e}_{j-1}\right\|_{\Phi_{j}}^{2},
\end{aligned}
$$

the first one depending on $v>1$, i.e. on the initial support radius and the second one depending on the mesh norm reduction rate $\mu<1$. Thus, assuming that the first term dominates the error, we can test 


\begin{tabular}{lllllll}
$N$ & $H$ & $\|\mathbf{e}\|_{\mathbf{L}_{2}(\Omega)}$ & $\|\mathbf{e}\|_{\mathbf{H}^{1}(\Omega)}$ & $\|\mathbf{e}\|_{\mathbf{L}_{\infty}(\Omega)}$ & $\operatorname{cond}(A)$ & ratio [\%] \\
\hline 9 & $2^{-1}$ & $1.83 \mathrm{e}-1$ & $1.53 \mathrm{e} 0$ & $6.11 \mathrm{e}-1$ & $1.3 \mathrm{e} 2$ & 72.22 \\
25 & $2^{-2}$ & $3.35 \mathrm{e}-2$ & $5.00 \mathrm{e}-1$ & $2.19 \mathrm{e}-1$ & $9.2 \mathrm{e} 2$ & 74.96 \\
81 & $2^{-3}$ & $5.62 \mathrm{e}-3$ & $1.65 \mathrm{e}-1$ & $6.19 \mathrm{e}-2$ & $5.2 \mathrm{e} 3$ & 52.44 \\
289 & $2^{-4}$ & $1.02 \mathrm{e}-3$ & $6.13 \mathrm{e}-2$ & $1.75 \mathrm{e}-2$ & $2.4 \mathrm{e} 4$ & 24.75 \\
1089 & $2^{-5}$ & $1.91 \mathrm{e}-4$ & $2.46 \mathrm{e}-2$ & $4.80 \mathrm{e}-3$ & $9.1 \mathrm{e} 4$ & 10.19 \\
4225 & $2^{-6}$ & $3.37 \mathrm{e}-5$ & $1.02 \mathrm{e}-2$ & $1.17 \mathrm{e}-3$ & $2.9 \mathrm{e} 5$ & 4.31 \\
16641 & $2^{-7}$ & $5.29 \mathrm{e}-6$ & $4.47 \mathrm{e}-3$ & $2.81 \mathrm{e}-4$ & $8.3 \mathrm{e} 5$ & 1.54 \\
\hline
\end{tabular}

Table 1: Convergence study for the multilevel interpolation of the divergence-free vector field (4.1) with basis function $\phi_{2,3}$ and support radii (4.2) where $v=2.5$.

\begin{tabular}{llllll}
$N$ & $H$ & $\|\mathbf{e}\|_{\mathbf{L}_{2}(\Omega)}$ & $\|\mathbf{e}\|_{\mathbf{H}^{1}(\Omega)}$ & $\|\mathbf{e}\|_{\mathbf{L}_{\infty}(\Omega)}$ & $\operatorname{cond}(A)$ \\
\hline 25 & $2^{-2}$ & 2.45 & 1.61 & 1.48 & -2.83 \\
81 & $2^{-3}$ & 2.57 & 1.60 & 1.83 & -2.50 \\
289 & $2^{-4}$ & 2.46 & 1.43 & 1.83 & -2.20 \\
1089 & $2^{-5}$ & 2.42 & 1.31 & 1.86 & -1.93 \\
4225 & $2^{-6}$ & 2.50 & 1.27 & 2.04 & -1.69 \\
16641 & $2^{-7}$ & 2.67 & 1.20 & 2.05 & -1.50 \\
\hline
\end{tabular}

Table 2: Orders for the multilevel interpolation of the divergence-free vector field (4.1) with basis function $\phi_{2,3}$ and support radii (4.2) where $v=2.5$.

this by increasing $v$ while leaving the rest of the setting unchanged. The results of this can be found in Table 5. They indicate that the determining term in (4.4) is indeed the first one, i.e. the one involving $v$. They also show that the best $L_{2}$-approximation order $\sigma=3.5$ can be achieved if the initial support radius is chosen sufficiently large. Finally, for this example, we also see that a good approximation can be achieved already with only a few levels (or data points) if the initial support is chosen sufficiently large.

\section{REFERENCES}

\begin{tabular}{lllllll}
$N$ & $H$ & $\|\mathbf{e}\|_{\mathbf{L}_{2}(\Omega)}$ & $\|\mathbf{e}\|_{\mathbf{H}^{1}(\Omega)}$ & $\|\mathbf{e}\|_{\mathbf{L}_{\infty}(\Omega)}$ & $\operatorname{cond}(A)$ & ratio [\%] \\
\hline 9 & $2^{-1}$ & $2.00 \mathrm{e}-1$ & $1.70 \mathrm{e} 0$ & $6.18 \mathrm{e}-1$ & $9.4 \mathrm{e} 1$ & 72.22 \\
25 & $2^{-2}$ & $4.10 \mathrm{e}-2$ & $6.11 \mathrm{e}-1$ & $2.72 \mathrm{e}-1$ & $2.3 \mathrm{e} 2$ & 58.00 \\
81 & $2^{-4}$ & $7.88 \mathrm{e}-3$ & $2.28 \mathrm{e}-1$ & $8.69 \mathrm{e}-2$ & $3.9 \mathrm{e} 2$ & 25.95 \\
289 & $2^{-4}$ & $1.68 \mathrm{e}-3$ & $9.77 \mathrm{e}-2$ & $2.56 \mathrm{e}-2$ & $5.2 \mathrm{e} 2$ & 8.75 \\
1089 & $2^{-5}$ & $5.44 \mathrm{e}-4$ & $6.22 \mathrm{e}-2$ & $7.00 \mathrm{e}-3$ & $6.1 \mathrm{e} 2$ & 2.55 \\
4225 & $2^{-6}$ & $3.90 \mathrm{e}-4$ & $8.54 \mathrm{e}-2$ & $1.62 \mathrm{e}-3$ & $6.7 \mathrm{e} 2$ & 0.69 \\
16641 & $2^{-7}$ & $4.15 \mathrm{e}-4$ & $1.66 \mathrm{e}-2$ & $9.85 \mathrm{e}-4$ & $6.6 \mathrm{e} 2$ & 0.18 \\
\hline
\end{tabular}

Table 3: Convergence study for the multilevel interpolation of divergence-free vector field (4.1) with basis function $\phi_{2,3}$ and support radii (4.3) where $v=2.5$. 


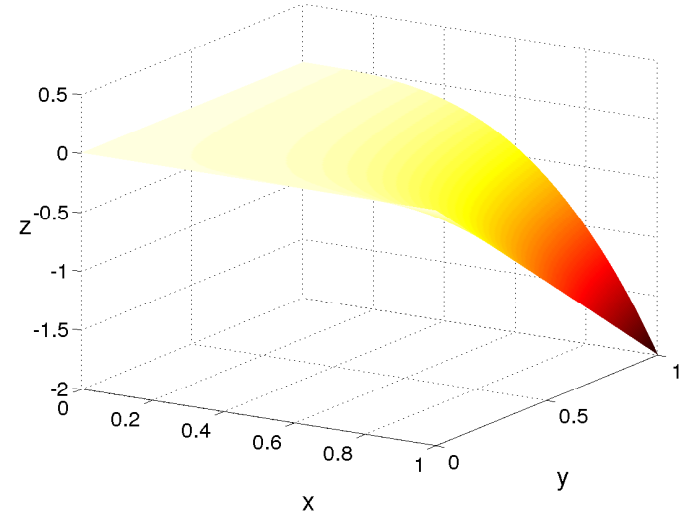

(a) $u_{1}$

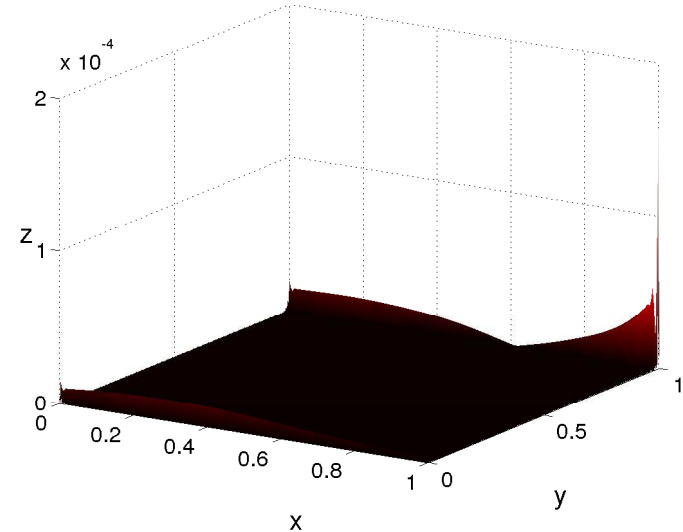

(c) $e_{1}$

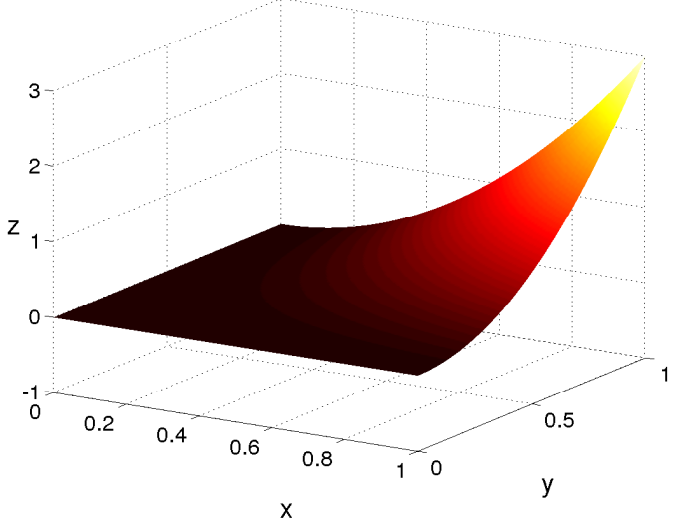

(b) $u_{2}$

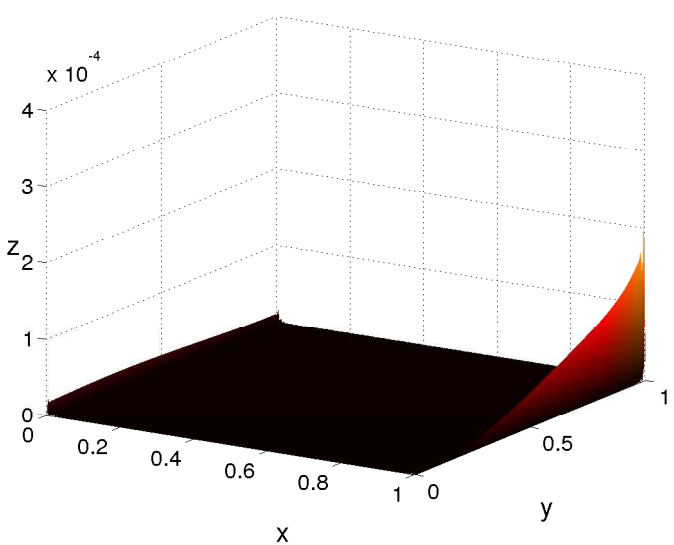

(d) $e_{2}$

FIG. 1: Both components of multilevel approximation and corresponding errors of divergence-free vector field (4.1) after seven levels, using the basis function $\phi_{2,3}, \mu=0.5$ and $v=2.5$. 


\begin{tabular}{llllll}
$N$ & $H$ & $\|\mathbf{e}\|_{\mathbf{L}_{2}(\Omega)}$ & $\|\mathbf{e}\|_{\mathbf{H}^{1}(\Omega)}$ & $\|\mathbf{e}\|_{\mathbf{L}_{\infty}(\Omega)}$ & $\operatorname{cond}(A)$ \\
\hline 25 & $2^{-2}$ & 2.28 & 1.47 & 1.18 & -1.29 \\
81 & $2^{-3}$ & 2.38 & 1.42 & 1.65 & -0.78 \\
289 & $2^{-4}$ & 2.23 & 1.22 & 1.76 & -0.41 \\
1089 & $2^{-5}$ & 1.63 & 0.65 & 1.87 & -0.23 \\
4225 & $2^{-6}$ & 0.48 & -0.45 & 2.11 & -0.12 \\
16641 & $2^{-7}$ & -0.09 & 2.37 & 0.72 & 0.02 \\
\hline
\end{tabular}

Table 4: Orders for the multilevel interpolation of the divergence-free vector field (4.1) with basis function $\phi_{2,3}$ and support radii (4.3) where $v=2.5$.

\begin{tabular}{l|ll|ll|ll} 
& \multicolumn{2}{|c|}{$v=2.5$} & \multicolumn{2}{c|}{$v=5$} & \multicolumn{2}{c}{$v=10$} \\
\hline$N$ & $\|\mathbf{e}\|_{\mathbf{L}_{2}(\Omega)}$ & order & $\|\mathbf{e}\|_{\mathbf{L}_{2}(\Omega)}$ & order & $\|\mathbf{e}\|_{\mathbf{L}_{2}(\Omega)}$ & order \\
\hline 9 & $1.83 \mathrm{e}-1$ & & $7.22 \mathrm{e}-2$ & & $5.61 \mathrm{e}-2$ & \\
25 & $3.35 \mathrm{e}-2$ & 2.45 & $9.11 \mathrm{e}-3$ & 2.99 & $5.19 \mathrm{e}-3$ & 3.43 \\
81 & $5.62 \mathrm{e}-3$ & 2.57 & $1.20 \mathrm{e}-3$ & 2.92 & $5.22 \mathrm{e}-4$ & 3.32 \\
289 & $1.02 \mathrm{e}-3$ & 2.46 & $1.65 \mathrm{e}-4$ & 2.87 & $5.55 \mathrm{e}-5$ & 3.23 \\
1089 & $1.91 \mathrm{e}-4$ & 2.42 & $2.30 \mathrm{e}-5$ & 2.84 & $5.83 \mathrm{e}-6$ & 3.25 \\
4225 & $3.37 \mathrm{e}-5$ & 2.50 & $3.06 \mathrm{e}-6$ & 2.91 & $5.87 \mathrm{e}-7$ & 3.31 \\
16641 & $5.29 \mathrm{e}-6$ & 2.67 & $3.58 \mathrm{e}-7$ & 3.10 & $5.26 \mathrm{e}-8$ & 3.48 \\
\hline
\end{tabular}

Table 5: Convergence study for the multilevel interpolation of the divergence-free vector field (4.1) with basis function $\phi_{2,3}$ and support radii (4.2) for various $v$. Expected order $\sigma=3.5$.

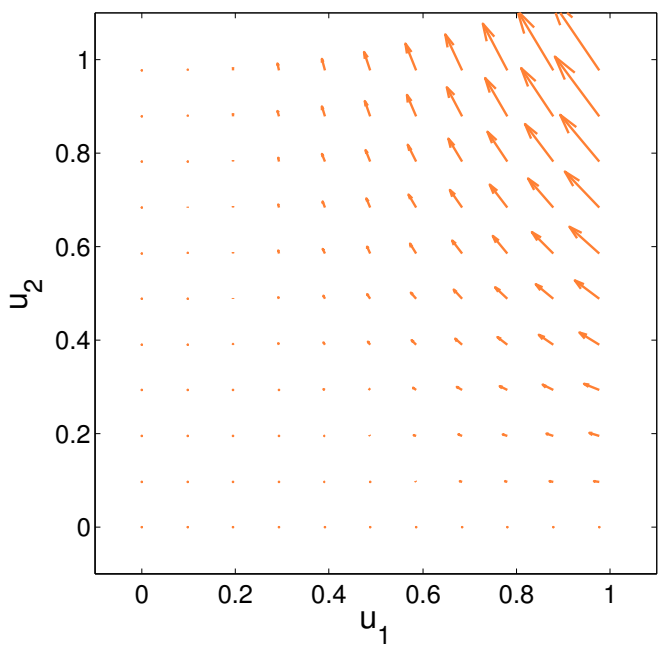

(a) Vector field $\mathbf{u}$

FIG. 2: The divergence-free vector field (4.1) after seven levels, using a basis function $\phi_{2,3}, \mu=0.5$ and $v=2.5$. 
BRACKBILL, J. \& BARNES, D. (1980) The effect of nonzero $\nabla \cdot B$ on the numerical solution of the magnetohydrodynamic equations. J. Comput. Phys., 35, 426-430.

Chernih, A. \& GiA, Q. T. L. (2013) Multiscale methods with compactly supported radial basis functions for the Stokes problem on bounded domains. Preprint University of New South Wales, Australia.

Evans, L. C. (1998) Partial Differential Equations, vol. 19. Providence: American Mathematical Society.

FARRell, P. \& Wendland, H. (2013) RBF multiscale collocation for second order elliptic boundary value problems. SIAM J. Numer. Anal., 51, 2403-2425.

FASSHAUER, G. E. (1999) Solving differential equations with radial basis functions: Multilevel methods and smoothing. Adv. Comput. Math., 11, 139-159.

FloATER, M. S. \& IsKe, A. (1996) Multistep scattered data interpolation using compactly supported radial basis functions. J. Comput. Appl. Math., 73, 65-78.

FlOATER, M. S. \& ISKE, A. (1998) Thinning algorithms for scattered data interpolation. BIT, 38, 705-720.

FUSELIER, E. (2008a) Erratum: Improved stability estimates and a characterization of the native space for matrixvalued RBFs. Adv. Comput. Math., 311-313.

FUSELIER, E. (2008b) Improved stability estimates and a characterization of the native space for matrix-valued RBFs. Adv. Comput. Math., 29, 269-290.

FUSELIER, E. (2008c) Sobolev-type approximation rates for divergence-free and curl-free RBF interpolants. Math. Comput., 77, 1407-1423.

Fuselier, E. J., NArcowich, F. J., Ward, J. D. \& Wright, G. B. (2009) Error and stability estimates for surface-divergence free RBF interpolants on the sphere. Math. Comput., 78, 2157-2186.

Johnson, M. J. (2012) Compactly supported, piecewise polyharmonic radial functions with prescribed regularity. Constr. Approx., 35, 201-223.

Le Gia, Q. T., Sloan, I. \& Wendland, H. (2010) Multiscale analysis in Sobolev spaces on the sphere. SIAM J. Numer. Anal., 48, 2065-2090.

LowitzSCH, S. (2005a) A density theorem for matrix-valued radial basis functions. Numer. Algorithms, 39 , 253-256.

Lowitzsch, S. (2005b) Error estimates for matrix-valued radial basis function interpolation. J. Approx. Theory, 137, 234-249.

LowitzSCH, S. (2005c) Matrix-valued radial basis functions: stability estimates and applications. Adv. Comput. Math., 23, 299-315.

MCNALLY, C. P. (2011) Divergence-free interpolation of vector fields from point values - exact $\nabla \cdot \mathrm{B}=0$ in numerical simulations. MNRAS Letters, 413, L76-L80.

Narcowich, F. J., Schaback, R. \& WARD, J. D. (1999) Multilevel interpolation and approximation. Appl. Comput. Harmon. Anal., 7, 243-261.

NARcowich, F. J., WARd, J. D. \& Wright, G. B. (2007) Divergence-free RBFs on surfaces. J. Fourier Anal. Appl., 13, 643-663.

NARCOWICH, F. J. \& WARD, J. D. (1994) Generalized Hermite interpolation via matrix-valued conditionally positive definite functions. Math. Comput., 63, 661-687.

Schaback, R. (2011) The missing Wendland functions. Adv. Comput. Math., 34, 67-81.

Townsend, A. \& Wendland, H. (2013) Multiscale analysis in sobolev spaces on bounded domains with zero boundary values. IMA J. Numer. Anal., 33, 1095-1114.

Wendland, H. (1995) Piecewise polynomial, positive definite and compactly supported radial functions of minimal degree. Adv. Comput. Math., 4, 389-396.

Wendland, H. (2005) Scattered Data Approximation. Cambridge Monographs on Applied and Computational Mathematics. Cambridge, UK: Cambridge University Press.

Wendland, H. (2009) Divergence-free kernel methods for approximating the Stokes problem. SIAM J. Numer. Anal., 47, 3158-3179.

Wendland, H. (2010) Multiscale analysis in Sobolev spaces on bounded domains. Numer. Math., 116, 493-517. 